\title{
Gene Mutation Analysis in Papillary Thyroid Carcinoma Using a Multi-Gene Panel in China
}

\author{
Qiang Wang ${ }^{1,2}$ \\ Ning Zhao' \\ Jun Zhang'
}

'Department of General Surgery, Beijing Friendship Hospital, Capital Medical University, Beijing, People's Republic of China; ${ }^{2}$ Department of Thyroid surgery, Shanxi Provincial People's Hospital Affiliated to Shanxi Medical University, Taiyuan, 030000, People's Republic of China

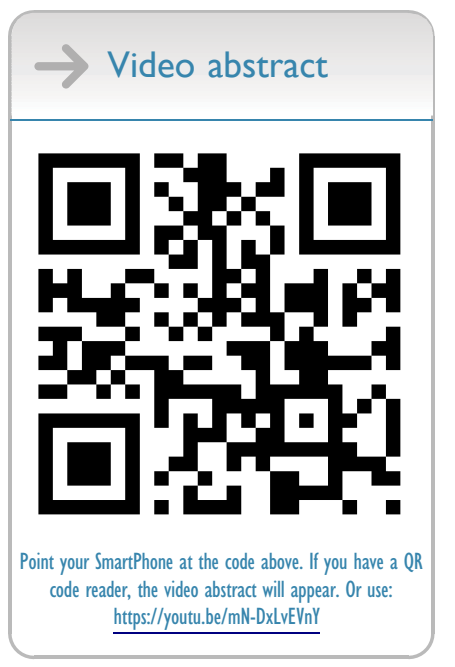

Correspondence: Jun Zhang Department of General Surgery, Beijing Friendship Hospital, Capital Medical University, Beijing, People's Republic of China

Tel +86 I38I I055986

Email zhangjun5986@ccmu.edu.cn
Purpose: To detect low-frequency mutation in the 57 genes of small panels that are associated with developing thyroid cancer in papillary thyroid carcinoma (PTC) patients and provide patients with precise-targeted therapy.

Patients and Methods: This study included 144 patients diagnosed with PTC who underwent total thyroidectomy and lymph node dissection in the central area of the neck between May 2017 and October 2018. We performed ultra-deep sequencing of 57 genes from 144 patients and detected the 57 genes mutations with bioinformatics.

Results: There were 698 mutations in 45 genes from 138 PTC patients. A high frequency of mutations was detected in the RBM10 gene (44\%) and TERT (43\%), and some hotspot mutations, such as RBM10:p.E119D and TERT:p.P112fs, were also found.

Conclusion: Ultra-deep sequencing of small gene panels can find some low-frequency mutation genes, which can provide targeted therapy for patients.

Keywords: papillary thyroid carcinoma, thyroid cancer, BRAF, gene panel

\section{Introduction}

Thyroid tumors are the most common malignant tumors of the endocrine system, and their incidence has been increasing in the recent decades. Currently, there are some target drugs that can effectively treat PTC, and next-generation sequencing (NGS) can be used for targeted therapy. In order to make better informed treatment decisions for patients with thyroid cancer, we need to detect the gene mutations, especially those associated with potential metastasis. Additionally, it is necessary to identify the different characteristics of oncogenes and tumor suppressor genes related to thyroid tumorigenesis and potential targeting. In the past, due to cost and several other factors, whole-exome sequencing was rarely used to detect thyroid cancer-related genes. In recent years, there have been a variety of multigene panels for thyroid tissue examination. ${ }^{1}$ Technological advances, including NGS methods, have made it possible to use tumor DNA to screen a large number of gene loci from existing clinical samples; the NGS panels can enhance the capture of gene information, and when detecting 2 or more genes, it is more cost-effective for first-generation sequencing. High-throughput massive parallel sequencing technology and bioinformatics can be used to screen for tumor activation and suppressor gene mutations, including point mutations, indels, and copy number aberrations. $^{2}$

PTC mutations include the MAPK pathway elements, such as the BRAF, HRAS, KRAS, and NRAS genes, and the most important tumor suppressor TP53 gene. ${ }^{3}$ 
Approximately one-third of the PTCs have the BRAF V600E mutation. In addition to BRAF mutations, PAX8/ PPARG and RET/PTC gene rearrangements are also common in PTC mutations. ${ }^{4-7}$ Thus, different treatment options would be needed for various mutations in the genes associated with PTC. Simultaneous detection of known driver genes and new driver genes, combined with histological findings, will provide more accurate clinical results. In addition, this method may have potential applications in the supplementary diagnosis of suspected thyroid disease.

In this study, we aimed to screen 57 genes mutations related to with developing thyroid cancer in 144 PTC patients in the Shanxi province, China.

\section{Materials and Methods}

This is a prospective study. We evaluated 144 patients diagnosed with PTC who underwent total thyroidectomy and lymph node dissection in the central area of the neck between May 2017 and October 2018. A total of 144 patients with PTC were included in this study, including 59 men and 85 women within the age range of 21-56 years, with an average age of $48.5 \pm 3.5$ years. We collect the patient's age, gender, lymph node metastasis status and BRAF V600E, TERT gene mutation status and other data to analyze the correlation between gene mutation and clinicopathological characteristics. This study was conducted in accordance with the Declaration of Helsinki.

We studied the genomic DNA of surgical samples of PTC. The samples were fresh tissues obtained from the operation. All surgically removed thyroid tissue samples were subjected to detailed histological examination. All patients were diagnosed with PTC. The study was approved by the Research Ethics Committee of Shanxi Provincial People's Hospital (NO.201992), and all patients provided written informed consent.
The freshly collected tissues were sent to Youxun for genetic testing, and the Illumina NextSeq 500 highthroughput sequencer (Beijing, China) was used for nextgeneration sequencing (NGS). The sequencing covered 57 genes (Table 1) closely related to thyroid cancer, including part of the exons and the relevant genes containing regions involving single base mutation, insertion or deletion of a small fragment, gene copy number variation, gene fusion, and other types of variation.

\section{Data Analysis}

Using the FastQC software, ${ }^{8}$ raw sequencing of data was done for quality control and low-quality sequences were removed to obtain high-quality sequences; the BWA software $^{9}$ was used to align high-quality sequences to the human reference genome (hg19), and Qualimap2 ${ }^{10}$ was used for QC (quality control) for alignment. The Mutect2 algorithm in GATK $4{ }^{11}$ was used to identify raw somatic mutations. To filter germline mutation, we filter out raw somatic mutation with a population frequency $>0.1$, the mutations in dbSNP. To filter false somatic mutations, we filter raw somatic mutation with a sequencing depth $<50$, and the number of mutation reads $<5$. The Oncotator ${ }^{12}$ software was used to annotate the mutations, and the mutual exclusion and co-mutation were analyzed with maftools. ${ }^{13}$ Search the target drug for thyroid cancer in the Civic database, ${ }^{14}$ and analyze the gene mutation in the Civic database.

\section{Results}

Analysis of the correlation between gene mutation and patients' clinicopathology. According to the type of gene mutation, it is divided into non-BRAF V600E single-gene mutation group, BRAF V600E single-gene mutation group, non-TERT single-gene mutation group, and TERT single-gene mutation group. The results of univariate

Table I List of Gene in the Panel

\begin{tabular}{|l|l|l|l|l|l|l|}
\hline AKTI & AKT2 & ALK & APC & ATM & AXINI \\
\hline CCDC6 & CDKNIB & CDKN2A & CDKN2B & CDKN2C & CHEK2 & CTNNBI \\
EGFR & EIFIAX & EML4 & ERBB2 & EZHI & FGFR2 & GNAS \\
HRAS & IDHI & KIT & KRAS & MEDI2 & MENI \\
NCOA4 & NCOR2 & NFI & NF2 & NOTCHI & NRAS & NTRKI \\
OFDI & PAX8 & PDGFRB & PIK3CA & PPARG & PRKARIA \\
PTEN & RACI & RBI & RBMI0 & RET & SPOP & SDGFRA \\
STRN & TERT & TFG & TG II \\
ZNFI48 & & & & TP53 & TPM3 \\
\hline
\end{tabular}


Table 2 Correlation Between BRAF Mutation and Patients' Clinicopathology

\begin{tabular}{|c|c|c|c|c|}
\hline Characteristics & $\begin{array}{l}\text { No Gene } \\
\text { Mutation } \\
(n=42)\end{array}$ & $\begin{array}{l}\text { BRAF V600E } \\
\text { Mutation } \\
(n=102)\end{array}$ & $\chi^{2} / F$ & $P$ \\
\hline Gender & & & 3.191 & 0.074 \\
\hline Male & 22 & 37 & & \\
\hline Female & 20 & 65 & & \\
\hline $\begin{array}{l}\text { Average } \\
\text { age(years) }\end{array}$ & & & 0.034 & 0.852 \\
\hline$\leq 45$ & 17 & 43 & & \\
\hline$>45$ & 25 & 59 & & \\
\hline Pathology(PTC) & & & 2.024 & 0.154 \\
\hline Non-PTMC & 18 & 57 & & \\
\hline PTMC & 24 & 45 & & \\
\hline Lesion location & & & 0.012 & 0.91 \\
\hline Unilateral & 33 & 81 & & \\
\hline Bilateral & 9 & 21 & & \\
\hline $\begin{array}{l}\text { Lymph node } \\
\text { metastasis }\end{array}$ & & & 0.013 & 0.909 \\
\hline Yes & 28 & 69 & & \\
\hline No & 14 & 33 & & \\
\hline
\end{tabular}

Table 3 Correlation Between TERT Mutation and Patients' Clinicopathology

\begin{tabular}{|c|c|c|c|c|}
\hline Characteristics & $\begin{array}{l}\text { No Gene } \\
\text { Mutation } \\
(n=75)\end{array}$ & $\begin{array}{l}\text { TERT } \\
\text { Mutation } \\
(n=69)\end{array}$ & $\chi^{2} / F$ & $P$ \\
\hline Gender & & & 1.231 & 0.2672 \\
\hline Male & 34 & 25 & & \\
\hline Female & 41 & 44 & & \\
\hline Average age(years) & & & 1.61 & 0.204 \\
\hline$\leq 45$ & 35 & 25 & & \\
\hline$>45$ & 40 & 44 & & \\
\hline Pathology(PTC) & & & 24.87 & 0 \\
\hline Non-PTMC & 54 & 21 & & \\
\hline PTMC & 21 & 48 & & \\
\hline Lesion location & & & 5.33 & 0.021 \\
\hline Unilateral & 65 & 49 & & \\
\hline Bilateral & 10 & 20 & & \\
\hline $\begin{array}{l}\text { Lymph node } \\
\text { metastasis }\end{array}$ & & & 0.804 & 0.369 \\
\hline Yes & 48 & 49 & & \\
\hline No & 27 & 20 & & \\
\hline
\end{tabular}

analysis showed that TERT mutation was related to Lesion location $(p=0.021)$, and it was related to Pathology $(p=0)$. See Tables 2 and 3 .

\section{Sequencing Overview}

The reads number of 144 samples ranged from 4,915,148 to $33,632,576$. The align rate of the samples reached more than $99 \%$. As the reads increased, the average coverage also increased (151X-3721X). The reads number and coverage relationship are shown in Figure 1. As the reads number increased, the coverage also increased significantly.

\section{Mutation Frequency Analysis}

The mutations identified by the Mutect2 software were filtered germline mutation and low-quality somatic mutation. Finally, a total of 114 exon regions of 57 genes were detected. Six hundred and eighty-nine mutations were detected at 314 position in 45 genes in 138 samples (6 samples were not detected mutation in 57 genes of this panel). The frequency of these mutations was unevenly distributed (0.11-99.84\%), as shown in Figure 2. There were 372 mutations with a frequency of $<1 \%$, accounting for $53.99 \%$ of the mutations, and 439 with $<5 \%$ frequency, accounting for $63.71 \%$. Most of these mutations were low frequency. These low-frequency mutation sites are difficult to detect by whole-genome sequencing or low-depth whole-exome sequencing $(<500 \mathrm{X})$. Using ultra-highdepth panel sequencing can help identify these lowfrequency sites. Since most mutations are of low

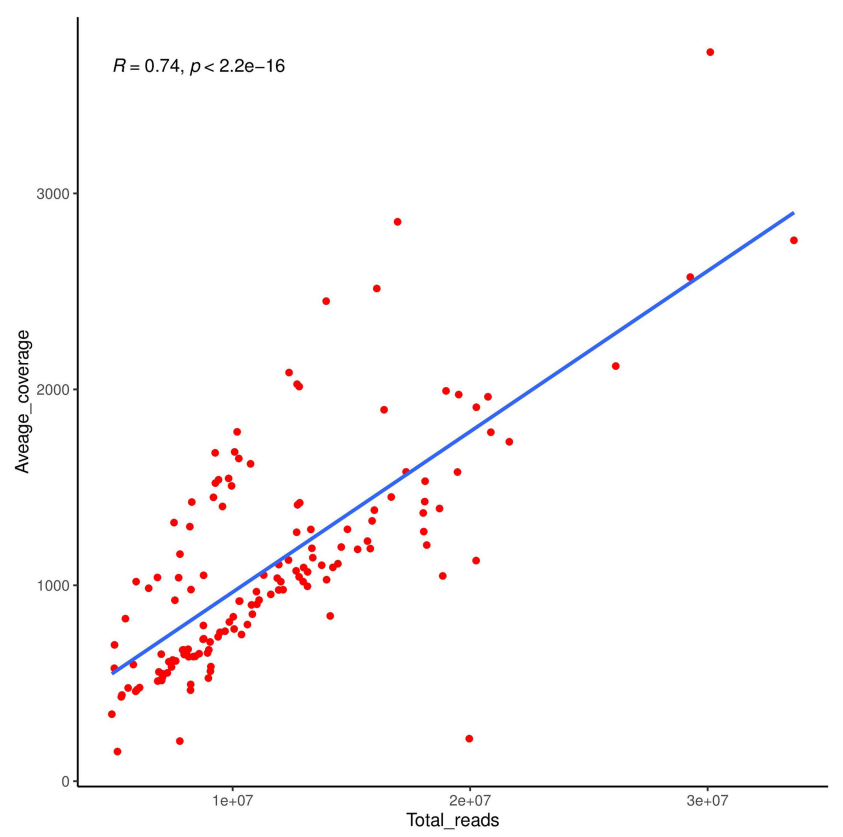

Figure I The reads number and coverage relationship, The $\mathrm{x}$-axis represents the reads number of samples, and the $y$-axis represents the average coverage of sample. 


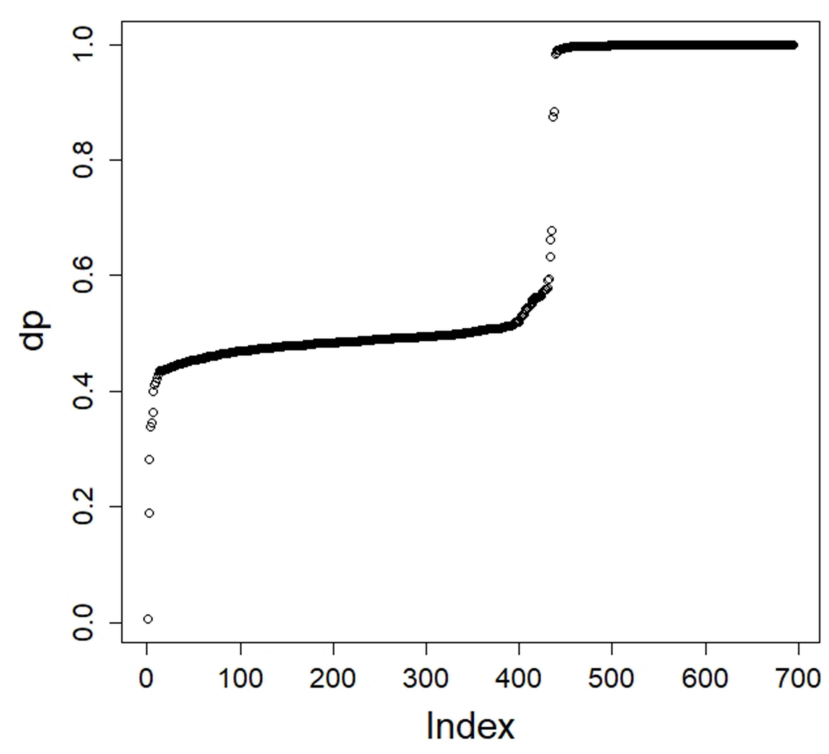

Figure 2 Mutation frequency distribution, $\mathrm{X}$-axis represents mutation, $\mathrm{Y}$ axis represents mutation frequency.

frequency, a Droplet Digital PCR (ddPCR) with high sensitivity is required for verification.

From the Civic database, it was found that 14 genes of thyroid cancer had relevant drug information, as shown in Table 4. The targeted drug site BRAF.p.V600E was found in 102 patients, in which the variant allele frequency (VAF) ranged from $0.245 \%$ to $40.27 \%$, among them there were 30 patients with a mutation frequency of $<0.5 \%$. For other targeted drug sites, we found that one patient was found to have an NRAS.p.Q61R mutation (11.81\%), and another patient was found to have an HRAS.p.G12C (3.2\%) mutation, which two patients can benefit from targeted drugs from the Civic database.

\section{Overview of Gene Mutations in the PTC Patients}

An overview of all mutations in 144 patients is shown in Figure 3. There were 138 samples mutated in the 57 genes of this panel. Mutations in the BRAF gene were detected in many samples. Mutations were found in up to $74 \%$ of the samples, see Figure 4 , and all of these were V600E mutations. The mutation frequency of these mutation sites is generally biased. The low-frequency mutations need further verification along with the RBM10 gene mutation, which was observed as a mutation hotspot RBM10: p.E119D (41.13\%) in this study, as shown in Figure 5. Finally, the TERT gene also showed mutations in $50 \%$ of the samples and was also observed as a mutation hotspot TERT: p.P112fs (37.59\%). Figure 6 shows the TERT mutation distribution.

\section{Mutually Exclusive and Co-Mutation Analysis}

We observed three pairs of mutually exclusive mutations in BRAF-CDKN2C ( $\mathrm{p}=0.01273)$, MED12-BRAF ( $\mathrm{p}=$ $0.0356)$, and BRAF-BRAF ( $p=0.0372)$. There were many co-mutated genes, with the most significant being the STK11 and AXIN1 genes $(p=6.81$ e -06$)$. See Figure 7.

\section{Discussion}

Although most PTCs can be successfully treated with radioiodide and levothyroxine suppression after complete surgical intervention, there is still a certain proportion of PTCs that are resistant to treatment and cause comorbidities and death. From an analysis at the molecular level, it has been further clarified that the pathogenesis of PTC is significantly related to thyroid invasion, advanced disease, lymph node metastasis, and tumor recurrence. A multigene panel can be used for gene analysis to understand the changes in tumor suppressor genes. ${ }^{15}$

Now, liquid biopsy can non-invasively reflect the status of tumors in the body, and provide a strong basis for early diagnosis of tumors, individualized treatment monitoring and prognostic judgment. According to the types of tumorrelated substances, liquid biopsy covers circulating tumor cells (CTCs), circulating tumor DNA (ctDNA), extracellular vesicles (EVs) and circulating tumor RNA (ctRNA) and other detection targets. ${ }^{16}$ The detection of thyroid cancer indicators through liquid biopsy technology can effectively assess the risk of thyroid cancer in the subject, so that they can receive more timely and effective treatment, so as to reduce the risk of thyroid cancer. At the same time, after routine examination and diagnosis, the patient can be diagnosed by liquid biopsy technology, and the tumor cell growth and development status and metastasis in the body can be evaluated and diagnosed to further confirm the specific condition of the patient. At the same time, the doctor can perform the diagnosis on the patient's body and understanding in many aspects, adjusting the diagnosis and treatment plan, and making relevant preventive diagnosis and treatment measures, so that the patient's condition can be effectively controlled.

At present, droplet digital PCR (ddPCR) is considered to be the preferred technique for detecting rare mutations in liquid biopsy samples. ${ }^{17}$ Recent studies have shown that ddPCR can 
Table 4 Thyroid Cancer-Related Targeted Drug Sites in the CIVIC Database

\begin{tabular}{|c|c|c|c|c|}
\hline Gene & Variant & Disease & Drugs & Evidence_Level \\
\hline RET & M918T & Thyroid Gland Medullary Carcinoma & & B \\
\hline RET & C634W & Thyroid Gland Medullary Carcinoma & Motesanib & $\mathrm{D}$ \\
\hline RET & M918T & Thyroid Gland Medullary Carcinoma & Motesanib & $\mathrm{D}$ \\
\hline RET & M918T & Thyroid Gland Medullary Carcinoma & JAK2 Inhibitor AZDI480 & $\mathrm{D}$ \\
\hline RET & M918T & Thyroid Gland Medullary Carcinoma & & B \\
\hline BRAF & V600E & Thyroid Gland Cancer & & B \\
\hline BRAF & V600E & Thyroid Gland Papillary Carcinoma & & B \\
\hline BRAF & V600E & Thyroid Gland Papillary Carcinoma & & B \\
\hline BRAF & V600E & Thyroid Gland Papillary Carcinoma & & B \\
\hline BRAF & V600E & Thyroid Gland Papillary Carcinoma & & B \\
\hline BRAF & V600E & Thyroid Gland Papillary Carcinoma & & B \\
\hline BRAF & AKAP9-BRAF & Thyroid Gland Papillary Carcinoma & & B \\
\hline NRAS & Q6I & Thyroid Gland Follicular Carcinoma & & B \\
\hline PAX8 & PAX8-PPARG & Thyroid Gland Follicular Carcinoma & & B \\
\hline TERT & $\mathrm{C} 228 \mathrm{~T}$ & Thyroid Gland Papillary Carcinoma & & B \\
\hline BRAF & V600E & Thyroid Gland Papillary Carcinoma & & B \\
\hline TERT & Promoter Mutation & Thyroid Gland Cancer & & B \\
\hline TERT & Promoter Mutation & Thyroid Gland Cancer & & B \\
\hline TSC2 & QII78* & Thyroid Gland Carcinoma & Everolimus & C \\
\hline MTOR & F2I08L & Thyroid Gland Carcinoma & Everolimus & C \\
\hline CD274 & Expression & Thyroid Gland Papillary Carcinoma & & B \\
\hline RET & M918T & Thyroid Gland Medullary Carcinoma & Sorafenib & B \\
\hline BRAF & $\mathrm{V} 600 \mathrm{E}$ & Thyroid Gland Papillary Carcinoma & Vemurafenib & C \\
\hline BRAF & V600E & Thyroid Gland Papillary Carcinoma & Vemurafenib & B \\
\hline PIK3CA & HI047R & Thyroid Gland Cancer & Perifosine, Temsirolimus & $\mathrm{D}$ \\
\hline PIK3CA & $\mathrm{E} 542 \mathrm{~K}$ & Thyroid Gland Cancer & Perifosine, Temsirolimus & $\mathrm{D}$ \\
\hline PTEN & $\mathrm{R} 130^{*}$ & Thyroid Gland Cancer & Perifosine, Temsirolimus & $\mathrm{D}$ \\
\hline TERT & $\mathrm{C} 228 \mathrm{~T}$ & Thyroid Gland Cancer & & B \\
\hline MENI & FRAMESHIFT TRUNCATION & Thyroid Gland Hurthle Cell Carcinoma & & C \\
\hline NF2 & Loss & Thyroid Gland Carcinoma & Selumetinib & $\mathrm{D}$ \\
\hline BRAF & V600E & Thyroid Gland Papillary Carcinoma & & B \\
\hline BRAF & V600E & Thyroid Gland Papillary Carcinoma & & B \\
\hline RET & C609Y & Thyroid Gland Medullary Carcinoma & & B \\
\hline GNAS & $\mathrm{R} 20 \mathrm{IH}$ & Thyroid Gland Follicular Carcinoma & Radioactive lodine & C \\
\hline ALK & Fusion & Thyroid Gland Anaplastic Carcinoma & Crizotinib & C \\
\hline BRAF & V600E & Thyroid Gland Anaplastic Carcinoma & Pertuzumab, Vemurafenib & C \\
\hline BRAF & $\mathrm{V} 600 \mathrm{E}$ & Thyroid Gland Anaplastic Carcinoma & Vemurafenib & B \\
\hline NTRK3 & ETV6-NTRK3 & Thyroid Gland Papillary Carcinoma & & B \\
\hline NRAS & Q6I & Thyroid Gland Follicular Carcinoma & & B \\
\hline HRAS & Q6I & Thyroid Gland Follicular Carcinoma & & B \\
\hline BRAF & V600E & Thyroid Gland Anaplastic Carcinoma & Trametinib, Dabrafenib & B \\
\hline RET & M918T & Thyroid Gland Medullary Carcinoma & Cabozantinib & B \\
\hline BRAF & $\mathrm{V} 600 \mathrm{E}$ & Thyroid Gland Papillary Carcinoma & Vemurafenib & B \\
\hline BRAF & V600E & Thyroid Gland Papillary Carcinoma & & B \\
\hline
\end{tabular}

be successfully used to monitor treatment response by quantifying BRAF and RAS mutants in cancer patient samples.

On the other hand, Co-amplification PCR (COLDPCR) based on lower denaturation temperature is an improved PCR method that allows the preferential amplification of rare mutant alleles within the target amplicon.
Recent studies have shown that the sensitivity of cold PCR to detect mutant variants is 10-100 times higher than that of standard PCR. ${ }^{18}$ At present, various versions of cold PCR have been developed and successfully used to detect mutant genes, including KRAS, HRAS, NRAS, EGFR, TP53 and BRAF. 


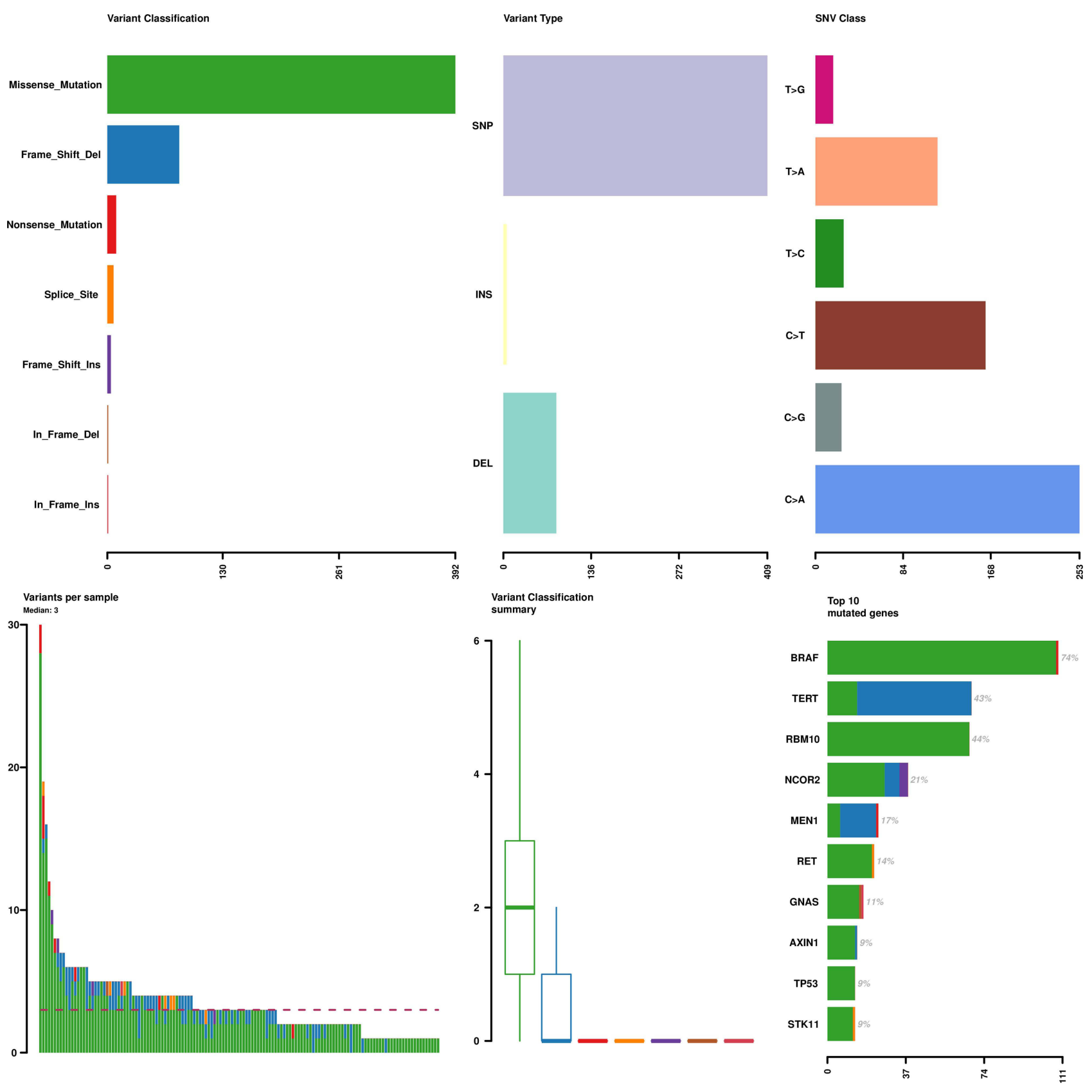

Figure 3 Mutation profile information. Upper Left: the number distribution of mutation classification; Upper Middle: the number distribution of mutation types and the number; Upper Right: distribution of SNV base mutations; Bottom Left: the distribution of mutation number of each sample; Bottom Middle: the number distribution of mutation classification; Bottom Right: the distribution of top 10 mutated genes.

The discovered mutations may have diagnostic and prognostic implications and help develop therapies for these potential molecular drivers. BRAF mutations are present in $45 \%$ to $80.8 \%{ }^{19-23}$ of PTCs and are related to regional metastasis and extrathyroidal expansion. Once thyroid cancer is highly suspected or diagnosed, risk factors must be considered, such as the clinical risk factors and tumor invasion behavior, patient's age and sex, initial size and location of the tumor, presence or absence of lymph nodes and/or distant metastasis, cytology and mutations data, and patient preferences. ${ }^{24} \mathrm{~A}$ positive BRAF mutation test indicates that the probability of malignancy is close to $100 \%$. This may help guide the scope of thyroidectomy.

Co-mutation has a synergistic effect on the occurrence and development of PTC and malignant biological behaviors, which can lead to increased tumor invasiveness, and can promote tumor progression to a certain extent, leading to poor prognosis. Co-mutation can be compared with mutations 


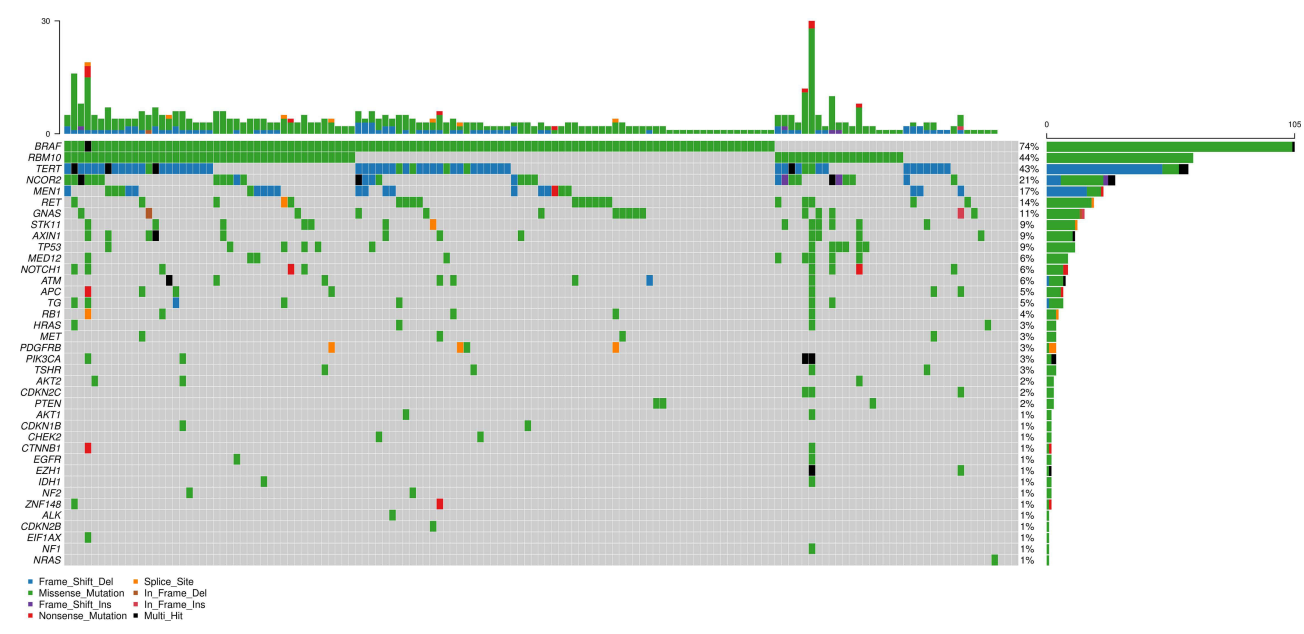

Figure 4 Mutation profile of 45 genes. The left side is the gene name, the right side is the gene mutation frequency, and the upper side is the number of gene mutations in the sample. Different colors indicate different mutation types.

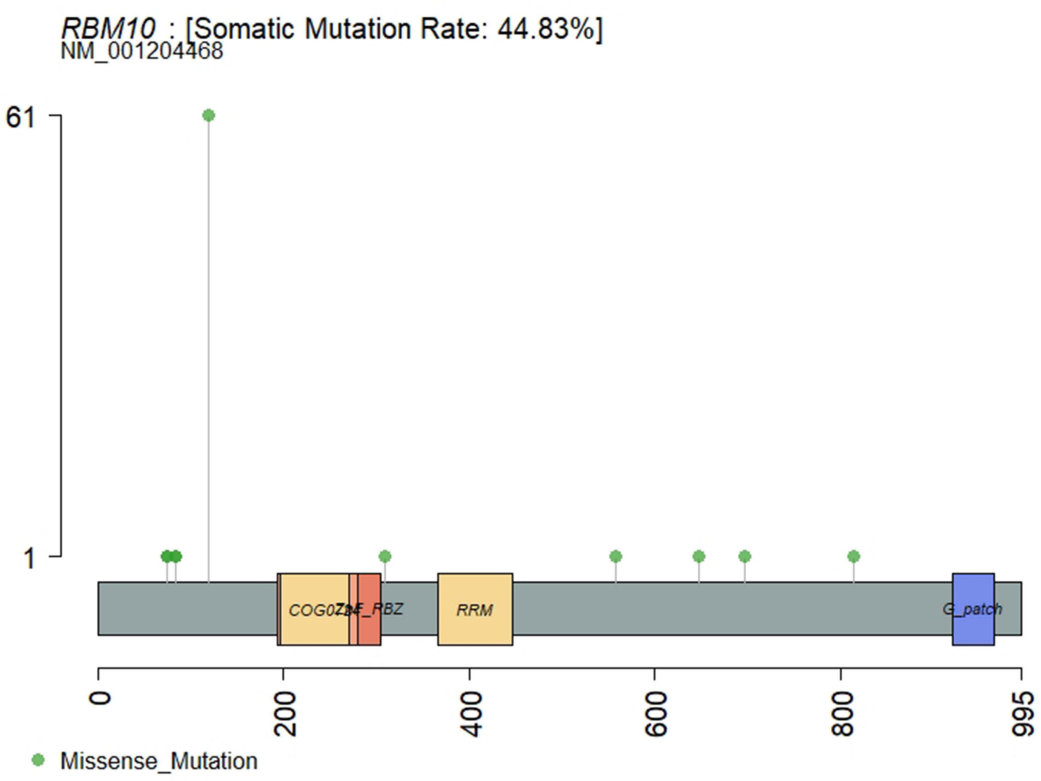

Figure 5 RBMI0 mutation distribution.

in a single gene. Make PTC more aggressive (prone to extrathyroid invasion, lymph node metastasis and distant metastasis), worse prognosis, recurrence and death. Co-mutation and exclusion analysis in human tumors are very important for cancer biology studies and treatment design.

In short, using small panel sequencing, genes can be ultra-deeply sequenced. In this study, the mutation coverage depth was $1000 \mathrm{X}$ on an average, and many ultralow-frequency mutations could be detected. Some lowfrequency mutations can provide clinicians with important targeted drug medication information. The most important
BRAF.p.V600E locus analyzed in this study had multiple mutations, all with very low frequency. Through sequencing of the small panel, we found that the frequency of many gene mutations is much higher than that reported in the previous studies. In previous studies of thyroid cancer, BRAF was the most significant mutation, with a mutation frequency of $56.9 \%$ in previous studies, which is higher in this study. It is possible that the use of ultra-deep sequencing may also be a characteristic of the Chinese population. In previous studies, the NRAS gene ranked second, with a mutation frequency of only $9.3 \% .{ }^{25}$ In this study, its 


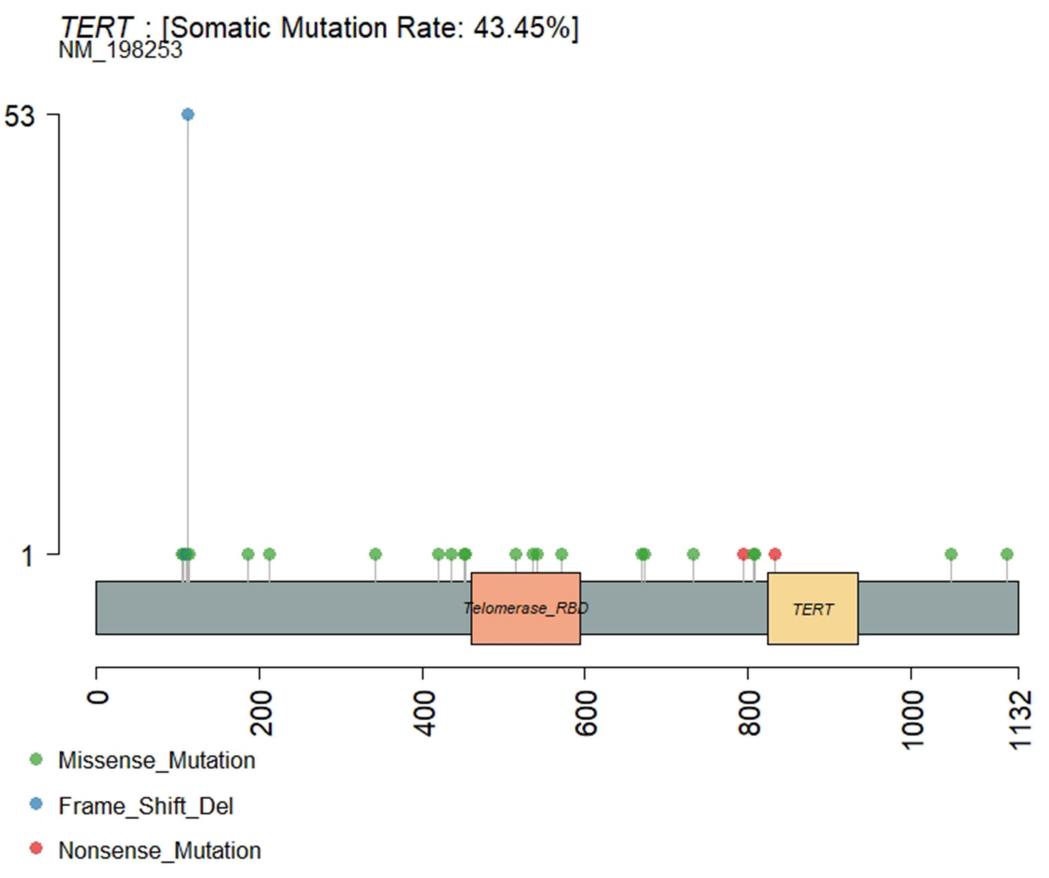

Figure 6 TERT mutation distribution.

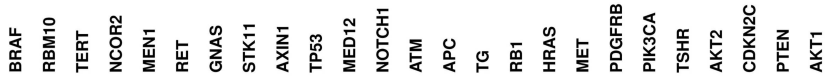

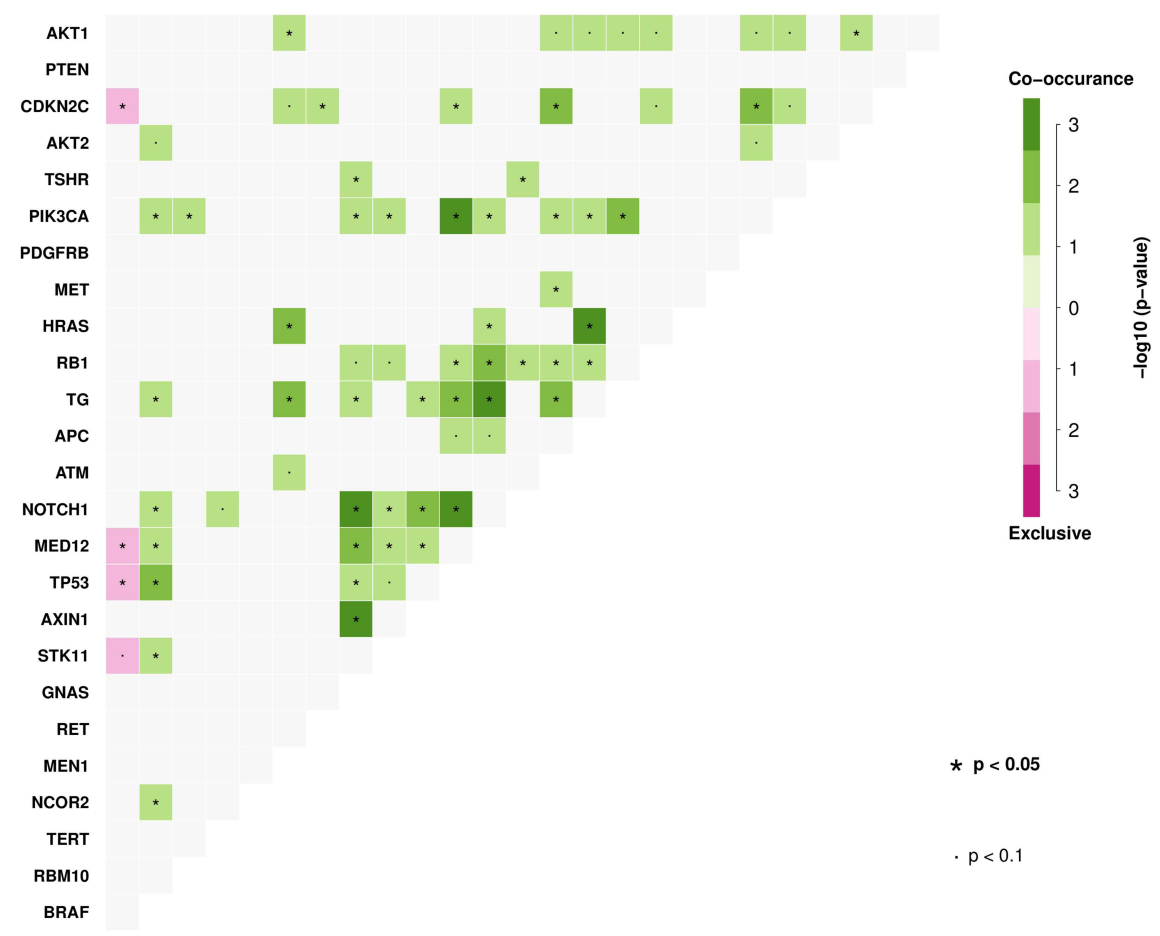

Figure 7 Overview of mutational mutual exclusion and co-mutation. *Means $\mathrm{p}<0.05$, Means $\mathrm{P}<0.1$. 
mutation frequency was only $1 \%$. However, the frequency of the second most mutated gene, RBM10, was as high as $44 \%$, which was reported as $0.3 \%$ in the previous studies. ${ }^{26}$ Moreover, some hotspot mutations, such as the RBM10: p.E119D (41.3\%), were found at low frequencies, and the highest mutation frequency of this locus was only $1.721 \%$. For mutations in this type of locus, verification needs to be done by ddPCR in a large number of samples. If this locus is a true positive site, it must be an important target for thyroid cancer, similar to the BRAF.p.V600E locus.

\section{Conclusion}

The diagnosis of uncertain thyroid lesions is a challenge in cytopathological practice. Some molecular markers, including the most studied BRAF and TERT promoter mutations, have been shown to have prognostic value. Panel sequencing can identify low-frequency mutations and detect additional mutations. In the era of targeted therapy, understanding the molecular characteristics of tumors is crucial for selecting the most appropriate anti-tumor drugs.

Panel sequencing can not only provide clinicians with more detailed references for targeted drug therapy but can also be very helpful for the investigation of driver genes in thyroid cancer.

\section{Acknowledgments}

We would like to thank the families who participated in this study.

\section{Disclosure}

The authors declare that they have no competing interests.

\section{References}

1. Nikiforova MN, Wald AI, Roy S, Durso MB, Nikiforov YE. Targeted next-generation sequencing panel (ThyroSeq) for detection of mutations in thyroid cancer. J Clin Endocrinol Metab. 2013;98(11):E1852E1860. doi:10.1210/jc.2013-2292

2. Poller DN, Glaysher S. Molecular pathology and thyroid FNA. Cytopathology. 2017;28(6):475-481. doi:10.1111/cyt.12492

3. Zhang K, Liu J, Li C, Peng X, Li H, Li Z. Identification and validation of potential target genes in papillary thyroid cancer. Eur J Pharmacol. 2019;843:217-225. doi:10.1016/j.ejphar.2018.11.026

4. Vuong HG, Kondo T, Oishi N, et al. Genetic alterations of differentiated thyroid carcinoma in iodine-rich and iodine-deficient countries. Cancer Med. 2016;5(8):1883-1889. PMID: 27264674; PMCID: PMC4898973. doi:10.1002/cam4.781

5. Nakazawa T, Kondo T, Kobayashi Y, et al. RET gene rearrangements (RET/PTC1 and RET/PTC3) in papillary thyroid carcinomas from an iodine-rich country (Japan). Cancer. 2005;104(5):943-951. PMID: 16015630. doi: $10.1002 / \mathrm{cncr} .21270$
6. Nikiforova MN, Biddinger PW, Caudill CM, Kroll TG, Nikiforov YE. PAX8-PPARgamma rearrangement in thyroid tumors: RT-PCR and immunohistochemical analyses. Am J Surg Pathol. 2002;26(8):1016-1023. PMID: 12170088. doi:10.1097/00000478200208000-00006

7. Armstrong MJ, Yang H, Yip L, et al. PAX8/PPAR $\gamma$ rearrangement in thyroid nodules predicts follicular-pattern carcinomas, in particular the encapsulated follicular variant of papillary carcinoma. Thyroid. 2014;24(9):1369-1374. PMID: 24798894; PMCID: PMC4148057. doi: 10.1089 /thy.2014.0067

8. Brown J, Pirrung M, McCue LA. FQC dashboard: integrates FastQC results into a web-based, interactive, and extensible FASTQ quality control tool. Bioinformatics. 2017;33(19):3137-3139. doi:10.1093/ bioinformatics/btx373

9. Li H, Durbin R. Fast and accurate long-read alignment with Burrows-Wheeler transform. Bioinformatics. 2010;26(5):589-595. doi:10.1093/bioinformatics/btp698

10. Okonechnikov K, Conesa A, García-Alcalde F. Qualimap 2: advanced multi-sample quality control for high-throughput sequencing data. Bioinformatics. 2016;32(2):292-294. doi:10.1093/bioinformatics/btv566

11. McKenna A, Hanna M, Banks E, et al. The genome analysis toolkit: a MapReduce framework for analyzing next-generation DNA sequencing data. Genome Res. 2010;20(9):1297-1303. doi:10.1101/ gr.107524.110

12. Ramos AH, Lichtenstein L, Gupta M, et al. Oncotator: cancer variant annotation tool. Hum Mutat. 2015;36(4):E2423-E2429. doi:10.1002/ humu. 22771

13. Mayakonda A, Lin DC, Assenov Y, Plass C, Koeffler HP. Maftools: efficient and comprehensive analysis of somatic variants in cancer. Genome Res. 2018;28(11):1747-1756. doi:10.1101/gr.239244.118

14. Griffith M, Spies NC, Krysiak K, et al. CIViC is a community knowledge base for expert crowdsourcing the clinical interpretation of variants in cancer. Nat Genet. 2017;49(2):170-174. doi:10.1038/ ng. 3774

15. Masoodi T, Siraj AK, Siraj S, et al. Evolution and impact of subclonal mutations in papillary thyroid cancer. Am J Hum Genet. 2019;105(5):959-973. doi:10.1016/j.ajhg.2019.09.026

16. Jensen K, Thakur S, Patel A, et al. Detection of BRAFV600E in liquid biopsy from patients with papillary thyroid cancer is associated with tumor aggressiveness and response to therapy. J Clin Med. 2020;9(8):2481. doi:10.3390/jcm9082481

17. Li X, Liu Y, Shi W, et al. Droplet digital PCR improved the EGFR mutation diagnosis with pleural fluid samples in non-small-cell lung cancer patients. Clin Chim Acta. 2017;471:177-184. doi:10.1016/j. cca.2017.06.007

18. Milbury CA, Correll M, Quackenbush J, Rubio R, Makrigiorgos GM. COLD-PCR enrichment of rare cancer mutations prior to targeted amplicon resequencing. Clin Chem. 2012;58(3):580-589. doi:10. 1373/clinchem.2011.176198

19. Lee SE, Hwang TS, Choi YL, et al. Molecular profiling of papillary thyroid carcinoma in Korea with a high prevalence of BRAFV600E mutation. Thyroid. 2017;27:802-810. doi:10.1089/ thy. 2016.0547

20. Tufano RP, Teixeira GV, Bishop J, et al. BRAF mutation in papillary thyroid cancer and its value in tailoring initial treatment. Medicine. 2012;91(5):274-286. doi:10.1097/MD.0b013e $31826 \mathrm{a} 9 \mathrm{c} 71$

21. Ke Z, Liu Y, Zhang Y, et al. Diagnostic value and lymph node metastasis prediction of a custom-made panel (thyroline) in thyroid cancer. Oncol Rep. 2018;40:659-668.

22. Hong AR, Lim JA, Kim TH, et al. The frequency and clinical implications of the BRAFV600EMutation in papillary thyroid cancer patients in Korea over the past two decades. Endocrinol Metab. 2014;29:505-513. doi:10.3803/EnM.2014.29.4.505 
23. Liang J, Cai W, Feng D, et al. Genetic landscape of papillary thyroid carcinoma in the Chinese population. J Pathol. 2018;244:215-226. doi:10.1002/path.5005

24. Fang Y, Ma X, Zeng J, et al. The profile of genetic mutations in papillary thyroid cancer detected by whole exome sequencing. Cell Physiol Biochem. 2018;50(1):169-178. doi:10.1159/000493966

25. Cabanillas ME, Dadu R, Iyer P, et al. Acquired secondary RAS mutation in BRAFV600E-mutated thyroid cancer patients treated with BRAF inhibitors. Thyroid. 2020;30(9):1288-1296. doi:10.1089/thy.2019.0514
26. Ibrahimpasic T, Xu B, Landa I, et al. Genomic alterations in fatal forms of non-anaplastic thyroid cancer: identification of MED12 and RBM10 as novel thyroid cancer genes associated with tumor virulence. Clin Cancer Res. 2017;23(19):5970-5980. doi:10.1158/ 1078-0432.CCR-17-1183

\section{Publish your work in this journal}

The International Journal of General Medicine is an international, peer-reviewed open-access journal that focuses on general and internal medicine, pathogenesis, epidemiology, diagnosis, monitoring and treatment protocols. The journal is characterized by the rapid reporting of reviews, original research and clinical studies across all disease areas. The manuscript management system is completely online and includes a very quick and fair peer-review system, which is all easy to use. Visit http://www.dovepress.com/ testimonials.php to read real quotes from published authors. 